\title{
Sirtuins, Aging, and Metabolism
}

\author{
LEONARD GuARENTE \\ Paul F. Glenn Laboratory and Department of Biology, Massachusetts Institute of Technology, \\ Cambridge, Massachusetts 02139 \\ Correspondence: leng@mit.edu
}

\begin{abstract}
Sirtuins are nicotinamide adenine dinucleotide (NAD)-dependent protein deacetylases that link protein acetylation, metabolism, aging, and diseases of aging. Sirtuins were initially found to slow aging in lower organisms and more recently shown to mediate many effects of calorie restriction on metabolism and longevity in mammals. This chapter focuses on two key mammalian sirtuins, SIRT1 (which resides mainly in the nucleus) and SIRT3 (which is mitochondrial). I discuss the many protein substrates of these sirtuins and how they determine the metabolic strategy most efficacious under scarce or abundant food supplies. I also discuss the logic by which sirtuins link protein acetylation and metabolism. Finally, I discuss emerging data showing protection by sirtuins against most of the common diseases of aging. It is possible that sirtuins will be novel targets to combat these diseases pharmacologically.
\end{abstract}

Sirtuins are NAD-dependent protein deacetylases that are homologous to yeast $\operatorname{Sir} 2 p$, one of the genes involved in silencing in that organism (Klar et al. 1979). Interest in sirtuins grew when Sir2 $p$ was shown to slow aging in yeast mother cells (Kaeberlein et al. 1999). Subsequent studies showed similar effects on aging in Caenorhabditis elegans and Drosophila (Tissenbaum and Guarente 2001; Rogina and Helfand 2004; Viswanathan et al. 2005; Berdichevsky et al. 2006; Bauer et al. 2009; Rizki et al. 2011; Viswanathan and Guarente 2011). Interest in sirtuins grew further when the yeast $\operatorname{Sir} 2 p$ and mammalian ortholog SIRT1 were shown to be NAD-dependent deacetylases (or class III deacetylases; Imai et al. 2000). In this unique reaction, NAD itself is cleaved every deacetylation cycle to generate $O$-acetyl ADPribose and nicotinamide, which are subsequently reassembled into NAD (Tanner et al. 2000).

Mammals have seven sirtuins, which are functionally nonredundant. SIRT1, SIRT6, and SIRT7 are primarily nuclear proteins, SIRT3, SIRT4, and SIRT5 are imported into mitochondria, and SIRT2 is cytoplasmic (Finkel et al. 2009; Haigis and Sinclair 2010; Verdin et al. 2010; Guarente 2011). Sirtuins execute their function by deacetylating many target proteins in the different cellular compartments. The physiological effects of deacetylation of sirtuin targets may be classified into two categories - the control of metabolism and the response to oxidative stress. In the first category, SIRT1 deacetylates many transcription factors that control the choice by cells of an oxidative versus glycolytic metabolic strategy, among them PGC- $1 \alpha$, HIF- $1 \alpha$, and $\operatorname{PPAR} \alpha$ (and other nuclear receptors; Rodgers et al. 2005; Purushotham et al. 2009). In addition, sirtuins deacetylate numerous metabolic enzymes to govern their specific activity. For example, SIRT1 and SIRT3 deacetylate the nuclear and mitochondrial isoforms of acetyl coenzyme-A (Co-A) synthetase to activate it by removing an acetyl group that blocks the active site (Hallows et al. 2006; Schwer et al. 2006). The sum of all of the known activities of sirtuins on transcription factors and metabolic enzymes would program cells for oxidative metabolism in mitochondria.

Sirtuins also help mediate the response to stress. SIRT1 deacetylates heat shock factor (HSF) to increase the perdurance of the heat shock response (Westerheide et al. 2009). Oxidative stress generates DNA damage, and SIRT1 and SIRT6 deacetylate numerous components of the DNA repair machinery, such as Ku70 and PARP (Haigis and Sinclair 2010; Nakagawa and Guarente 2011). Transcriptional targets of SIRT1, such as p53 and FOXO, also govern the cellular timing that balances repair versus apoptosis during the damage response. In addition, SIRT3 plays a major role in suppressing reactive oxygen species (ROS) in mitochondria, the primary source of oxidative stress, by deacetylating antioxidant enzymes and components of the electron transport chain itself (Bell and Guarente 2011). Finally, SIRT1 and SIRT6 both suppress another manifestation of ROS, the proinflammatory response, by suppressing the activity of the key regulator of inflammation, NF-kB. SIRT1 deactylates p65 of NF- $\kappa B$ to suppress its ability to activate proinflammatory transcriptional targets (Yeung et al. 2004), and SIRT6 deacetylates histones at the promoters of NF-kB-regulated genes to further suppress the proinflammatory output (Kawahara et al. 2009).

\section{SIRTUINS LINK PROTEIN ACETYLATION AND METABOLISM}

A large number of cellular proteins are acetylated. For example, recent studies have demonstrated that a high fraction of mitochondrial proteins are acetylated (Kim 
et al. 2006) as well as most metabolic enzymes in the cytosolic/nuclear pool (Wang et al. 2010; Zhao et al. 2010). Acetylation in the cytosol is carried out by histone acetyltransferases (HATs) and deacetylation by class I and II histone deacetylases (HDACs) as well as sirtuins. Among the sirtuins, SIRT1 appears to have the broadest range of substrates and affect the greatest number of physiological pathways (Finkel et al. 2009; Haigis and Sinclair 2010; Imai and Guarente 2010). In the mitochondria, the source of protein acetylation is not known (and may not even be enzymatic), but the most prominent deacetylase appears to be the sirtuin SIRT3 (Lombard et al. 2007).

Cells have two metabolic strategies to produce ATP, glycolysis, which takes place in the cytosolic/nuclear location, and oxidative phosphorylation, which occurs in the mitochondria. When energy is abundant in the diet, growth factors drive glucose uptake and activation of glycolysis. Thus, ATP is generated and NAD is converted to NADH (reduced NAD) in the cytosolic/nuclear pool (Fig. 1A). Because the sirtuins are NAD-dependent deacetylases, a high flux through glycolysis will lower NAD levels and thus repress the activity of SIRT1 as well as the other sirtuins in the nucleus and cytoplasm. Indeed, many studies have shown that SIRT1 activity is low in growth conditions of glucose excess and high during energy limitation (Finkel et al. 2009; Haigis and Sinclair 2010; Imai and Guarente 2010). Low SIRT1 activity would increase the acetylation status of many metabolic enzymes in the cytosolic/nuclear compartment, as long as the activities of HATs and HDACs were constant under changing energy supplies.
Indeed, in Salmonella, the acetylation of many glycolytic enzymes was shown to be higher in glucose-grown cells than in cells grown on a nonfermentable carbon source (Wang et al. 2010). This difference was at least partly mediated by the bacterial sirtuin cobB, because acetylation of these enzymes appeared to be higher in cobB mutants. One enzyme regulated in this way is glyceraldehyde-3-phosphate dehydrogenase (GAPDH), which converts glyceraldehyde 3-phosphate to 1,3-bisphosphoglycerate in glycolysis and catalyzes the reverse reaction in gluconeogenesis (a rare example in which the same enzyme functions at the same step of glycolysis and gluconeogenesis). Remarkably, acetylated GAPDH is better at driving the forward reaction used in glycolysis, whereas deacetylated GAPDH is more effective at the reverse reaction used in gluconeogenesis.

These simple findings suggest an autocatalytic, feedforward mechanism - diets rich in carbohydrate energy drive glycolysis, convert NAD to NADH, inactivate sirtuins, and increase acetylation and activity of GAPDH and perhaps other glycolytic enzymes (Fig. 1A). Intriguingly, mammalian GAPDH is also acetylated, and it will be important to determine whether it, as well as other acetylated metabolic enzymes in mammalian cells, is a SIRT1 substrate.

It is informative to follow the flow of carbon processed in glycolysis. The pyruvate produced by glycolysis has two possible fates. First, it can be reduced to fermentation products (bacteria), ethanol (yeast), or lactate (mammals), thereby converting some of the NADH produced in glycolysis back to NAD. Second, it can be used to
A
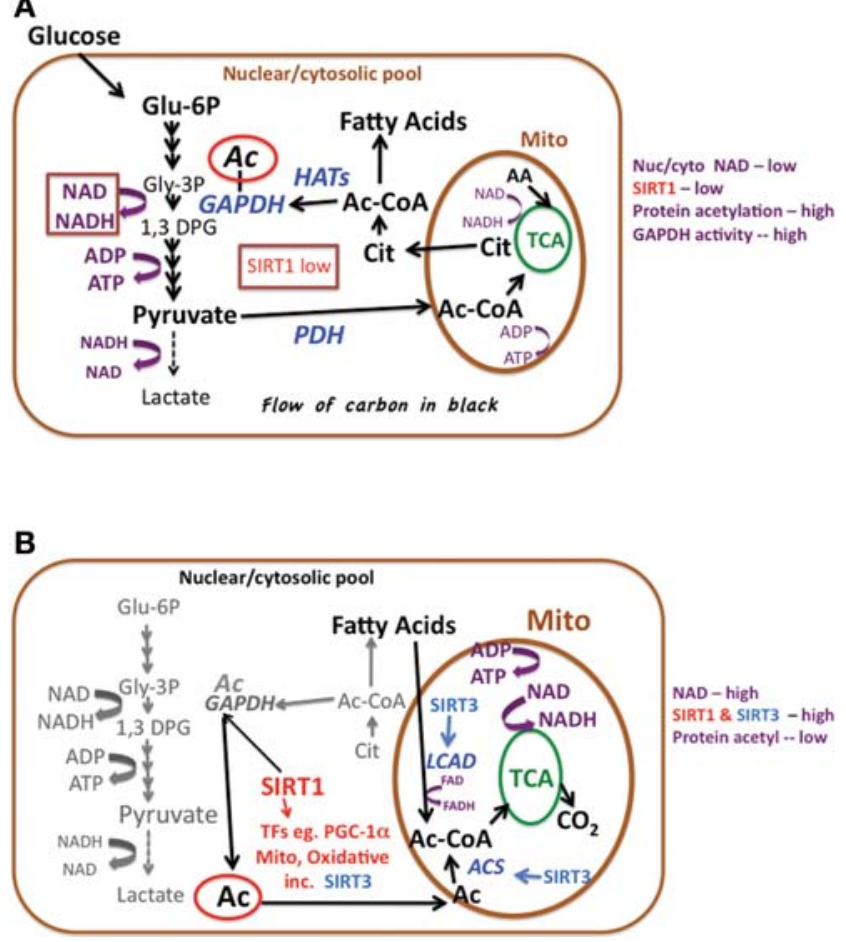

Figure 1. Linking protein acetylation, metabolism, and sirtuins. (A) ATP production by glycolysis and flow of carbon (black text and arrows) in energy storage tissues when energy is in excess. Mitochondria (Mito) and the tricarboxylic acid (TCA) cycle are indicated, and the remainder of the cell represents the cytosolic and nuclear compartments combined. Enzymes in blue italics are glyceraldehyde-3-phosphate dehydrogenase (GAPDH), histone acetyltransferases (HATs), pyruvate dehydrogenase (PDH), and ATP-citrate lyase (ACL). Note a feed-forward loop of high glycolysis and NADH production, low SIRT1 activity, and high acetylation and activity of glycolytic enzymes (e.g., GAPDH). Carbon flows as follows. Glucose 6-phosphate (Glu-6P) is converted to pyruvate, which can be reduced to lactate or converted to acetyl$\mathrm{CoA}$ (Ac-CoA) by PDH. In this case, citrate from the TCA cycle is transported to the cytosol and converted to Ac-CoA for fatty acid synthesis by ACL. Depletion of citrate from the TCA cycle necessitates anapleurotic conversion of amino acids (AA) to $\alpha$-ketogluterate to replenish the cycle. $(B)$ ATP production by oxidative metabolism and flow of carbon (black text and arrows) in energy-producing tissues upon energy deprivation. Glycolytic feedforward loop in $A$ is reversed, thereby activating SIRT1 and deacetylating and inhibiting glycolytic enzymes. SIRT1 generates $O$-acetyl ADP ribose and thus acetate (Ac) by deacetylating glycolytic proteins and induces mitochondrial biogenesis and $\beta$-oxidation of fatty acids by deacetylating transcription factors (TFs) such as PGC-1 $\alpha$. SIRT3 is also induced under energy limitation. Note that deacetylation of ACS and long-chain acyl dehydrogenase (LCAD) by SIRT3 in the mitochondria is known to activate those enzymes to generate acetyl-CoA. 
synthesize fatty acids as a primary mechanism of cell growth and energy storage. In this case, the pyruvate is converted into acetyl-CoA by pyruvate dehydrogenase $(\mathrm{PDH})$ in mitochondria and then re-derived back into cytoplasmic acetyl-CoA (Fig. 1A). This step occurs by transport of citrate from mitochondria to the cytosol, where ATP-citrate lyase (ACL) converts it to acetyl-CoA. ACL was recently shown to be required for normal histone acetylation in mammalian cells (Wellen et al. 2009), suggesting that the primary pathway of storing energy as fat may also provide the carbon for protein acetylation in the cytosolic/nuclear pool. By analogy with fatty acid synthesis, the high degree of protein acetylation in the cytosolic/nuclear pool during energy excess can be seen as another mechanism of storing carbon energy in dietary-sufficient conditions.

A reverse set of considerations applies to diets poor in energy: low glycolysis, activated SIRT1, and protein deacetylation in the cytosolic/nuclear pool (Fig. 1B). These conditions would perfectly match one of the main outputs of SIRT1 activity, the deacetylation of PGC- $1 \alpha$ to promote mitochondrial biogenesis and oxidative metabolism (Rodgers et al. 2005). Oxidative phosphorylation produces much more ATP per input glucose than fermentation, because the fuel is oxidized all the way to $\mathrm{CO}_{2}$. Because fatty acids are oxidized in mitochondria under these conditions, acetyl-CoA (and FADH) will be generated in this compartment. At the same time, the generation of acetylCoA in the cytoplasm by ATP-citrate lyase will be limited. Both the high SIRT1 activity and low cytosolic acetyl-CoA levels would favor maintenance of cytosolic/nuclear proteins in a relatively deacetylated state during energy-poor diets.

These considerations do not apply in any obvious way to mitochondrial protein acetylation. Indeed, changes in the acetylation of mitochondrial proteins with diet vary from tissue to tissue and are likely be distinct from acetylation of proteins in the nuclear/cytoplasmic pool (Schwer et al. 2009). There are likely to be competing processes affecting protein acetylation in this organelle. On the one hand, oxidative metabolism in mitochondria, for example, fatty acid oxidation, may be expected to increase acetyl-CoA levels in the organelle and thereby favor protein acetylation, perhaps occurring chemically (Fig. 1B). On the other, one of the mitochondrial proteins activated under these conditions is the major mitochondrial deacetylase SIRT3. Two key outputs of deacetylation by SIRT3 are to activate mitochondrial metabolic enzymes for oxidative metabolism and enzymes for detoxification of ROS (Verdin et al. 2010; Bell and Guarente 2011).

Protein acetylation serves as a regulatory mechanism, as well as an energy storage mechanism, at least in the nuclear/cytosolic compartment. The regulatory aspects minimally include histone acetylation to regulate gene transcription, acetylation of metabolic enzymes to favor glycolysis for ATP production, and acetylation of many transcription factors, such as p53, FOXO, PGC-1 $\alpha$, nuclear receptors, etc., to regulate expession levels of pathways for oxidative versus glycolytic metabolism
(Finkel et al. 2009; Haigis and Sinclair 2010; Imai and Guarente 2010). It is worth noting that the acetylation of lysines that are SIRT1 substrates can either be activating (e.g., p53) or repressing (e.g., PGC-1 $\alpha$ ), which has evidently allowed evolution greater flexibility in tinkering with the many affected pathways.

The idea that protein acetylation is a storage mechanism is most easily appreciated in cells transitioning from energy excess to energy limitation (Fig. 1B). Under these conditions, acetate generated by SIRT1 deacetylation of many proteins would be a substrate for mitochondrial ACS. Along with the oxidation of fatty acids, this would drive the TCA cycle and oxidative phosphorylation to yield ATP and $\mathrm{CO}_{2}$. It is rather striking that SIRT3, which is up-regulated during this energy transition, deacetylates and activates both the mitochondrial ACS and long-chain acyl dehydrogenase (LCAD) in the fatty acid oxidation pathway.

In the above model, protein acetylation governs how cells navigate between glycolytic and oxidative metabolism as a function of available energy. Because the substrates for protein acetylation are NAD and acetyl-CoA, it is not surprising that SIRT1, an NAD-dependent deacetylase, would be a central metabolic sensor and mediator of this process. The protein acetylation cycle depicted in the figure is interdigitated with other biological processes that affect the metabolic strategy of cells. For example, PARP-1 inhibition drove SIRT1 activity and oxidative metabolism by raising NAD levels (Bai et al. 2011). In addition, mice knocked out for the plasma membrane citrate transporter, INDY, showed metabolic effects similar to SIRT1 gain-of-function animals (Birkenfield et al. 2011). It will be very interesting to see whether these mice show decreased cytosolic acetyl-CoA (owing to decreased citrate in the cytosol), decreased protein acetylation, and decreased glycolysis - a pattern that is predicted to up-regulate SIRT1, as discussed in the model above.

\section{SIRTUINS MEDIATE CALORIE RESTRICTION}

Sir2p was shown to mediate extension of life span by modest glucose reduction in yeast. These findings were subsequently challenged in studies using a protocol with still lower glucose concentration in the media. In C. elegans and Drosophila, studies using different protocols have produced varying results that show dependence on sirtuins in some cases but not in others. In mice, calorie restriction $(\mathrm{CR})$ is much better defined than in the lower organisms $-60 \%-70 \%$ of ad libitum feeding. Here, many lines of evidence point to the critical nature of sirtuins in CR.

1. Sirtuin targets, including those mentioned above, are critical in the adaptation from energy excess (ad libitum feeding) to CR. This transition triggers an up-regulation of oxidative metabolism. For example, SIRT1 and SIRT3 were shown to activate genes encoding fatty acid oxidation by targeting PPAR $\alpha$ in the nucleus (SIRT1; Purushotham et al. 2009) and metabolic 
enzymes themselves in the fatty acid degradation pathway in the mitochondria (SIRT3; Hirschey et al. 2010). SIRT3, SIRT4, and SIRT5 were also shown to regulate other mitochondrial enzymes critical for the metabolic adaptation to energy limitation, such as glutamate dehydrogenase (Haigis et al. 2006) and enzymes of the urea cycle (Nakagawa et al. 2009; Hallows et al. 2011).

2. Levels of both SIRT1 and SIRT3 have been shown to rise in several tissues during CR (Cohen et al. 2004; Haigis and Sinclair 2010). This increase may, at least in part, be due to an increase in available NAD in the nuclear pool, concomitant to a lowering in the flux through glycolysis (which reduces NAD to NADH). In muscle and white adipose tissues, this increase has been directly demonstrated (Chen et al. 2008). It was recently shown that SIRT1 activates its own expression (Xiong et al. 2011), and it has been suggested that SIRT1 may also activate SIRT3 expression (Bell and Guarente 2011). Thus, an increase in NAD could elevate the protein levels of both of these key sirtuins.

3. Loss-of-function genetic evidence shows the importance of sirtuins in CR. Deletion of SIRT1 prevented outputs of CR, such as increased physical activity and extension in life span (Chen et al. 2005; Boily et al. 2008). Brain-specific SIRT1 deletion abolished the normal regulation of the somatotropic axis by diet, which emanates from the hypothalamus (Cohen et al. 2009). Deletion of any of the mitochondrial sirtuins resulted in metabolic defects in calorie limitation, such as increased blood ammonia (Nakagawa et al. 2009). Strikingly, deletion of SIRT3 has been shown to abolish the strong protective effect of CR against hearing loss in normal aging mice (Someya et al. 2010). This last example ties together major theses in aging research: oxidative damage, CR, sirtuins, and mitochondria.

4. Gain-of-function genetic evidence also implicates SIRT1 in CR. SIRT1 transgenic mice showed protection against metabolic disease with normal aging, mirroring effects of $\mathrm{CR}$ on wild-type animals (Bordone et al. 2007; Banks et al. 2008; Pfluger et al. 2008; Herranz et al. 2010). Importantly, these mice were also protected against diabetes, liver steatosis, bone loss, and general inflammation caused by obesity. Deacetylation of SIRT1 targets is evidently required for these effects, because a SIRT1 transgene with a catalytic mutation did not confer benefits.

5. Small-molecule SIRT1 activators have similar metabolic benefits to the SIRT1 transgenes (Baur et al. 2006; Lagogue et al. 2006). These activators include the natural product resveratrol (Howitz et al. 2003) and newer more potent synthetic compounds (Milne et al. 2007). In the latter case, efficacy was also demonstrated in a genetic model of murine obesity $(\mathrm{ob} / \mathrm{ob}$ mice; leptin deficient) and in obese rats. Some have argued that these compounds do not target SIRT1 (Pacholec et al. 2010), but the most recent report showed direct activation of this sirtuin by the newer chemicals in vitro (Dai et al. 2010). Consistent with the idea that the compounds may activate sirtuins and thus function as CR mimetics, transcriptional profiling showed a significant overlap between mice on CR and mice dosed with putative SIRT1-activating compounds (Barger et al. 2008). Finally, at least some of the effects of these compounds were abolished in mice in which SIRT1 had been deleted (Boily et al. 2009; Minor et al. 2011). Given the interest in this area, these compounds are now being studied intensely and their mechanism(s) of action should be worked out soon.

\section{SIRTUINS SLOW AGING AND DISEASES}

\section{SIRT1 and Metabolic Diseases}

An early indication of the potential importance of SIRT1 in metabolic diseases was the finding that the protein could influence differentiation and fat accumulation in 3T3-L1 cells and primary rat pre-adipocytes (Picard et al. 2004). In addition, SIRT1 was shown to deacetylate and activate peroxisome proliferator-activated receptor-g coactivator PGC-1 $\alpha$ (Rodgers et al. 2005), and CR triggered a SIRT1/PGC-1 $\alpha$-dependent increase in muscle mitochondrial biogenesis (Nisoli et al. 2005). Limiting calories also caused SIRT1 to deacetylate and activate peroxisome proliferator-activated receptor $\operatorname{PPAR} \alpha$ to turn on genes for fatty acid oxidation (Purushotham et al. 2009). In toto, these effects would favor oxidative metabolism in muscle, adipose tissues, and liver, insulin sensitivity, and perhaps a slower rate of aging-related decline.

In the fasted liver, SIRT1 governed two pathways to switch the way gluconeogenesis is regulated (Liu et al. 2008). Early after fasting, gluconeogenesis is activated by CREB and its coactivator CRTC2. The SIRT1 gene itself is also turned on by CREB/CRTC2 during early fasting via a site in its promoter (Noriega et al. 2011). The increase in SIRT1 then triggers deacetylation, ubiquitination, and destabilizion of CRTC2, resulting in a lowering of CREB activity. At the same time, SIRT1 deacetylates and activates PGC- $1 \alpha$ and FOXO to facilitate an alternate pathway of activation of genes for gluconeogenesis during more prolonged fasting. It is likely that a lower magnitude of activation occurs during these later times, when ketone bodies also help bridge the energy deficit. This second mechanism may also play an important role during CR.

SIRT1 transgenic mice can show CR-like phenotypes, as discussed above. These mice were also protected against an inflammation-induced model of liver cancer (Herranz et al. 2010). The anti-inflammatory effect of sirtuins may constitute a very important function, because both SIRT1 and SIRT6 repress NF-кB activity, as discussed above. Additional murine studies indicated that SIRT1 can be protective against colon cancer (Firestein et al. 2008) and breast cancer (Wang et al. 2008). Other studies suggest that this SIRT1 may also have oncogenic 
properties in certain contexts, so the relationship between SIRT1 and cancer may not be fully understood.

\section{SIRT1 and Neurodegenerative Diseases}

Neurodegenerative diseases have become a sobering obstacle to healthy aging in humans. It is estimated that Alzheimer's disease (AD) alone will affect up to onethird of those past age 80 . Methods to detect early stages of $\mathrm{AD}$ have become ever more sensitive, but treatments remain very elusive. Can sirtuins protect the brain? Neuronal stress, for example, in cultured neurons (Qin et al. 2006) or in the cyclin-dependent kinase-5 (cdk5) (p25) transgenic mice (Kim et al. 2007), could be mitigated by overexpression of SIRT1.

More relevant, in mice that overexpress two human genes that predispose to early AD, SIRT1 overexpression in the brain reduced the load of $A \beta$-amyloid peptide, the toxic agent in AD (Donmez et al. 2010). A $\beta$ is generated by sequential proteolytic cleavages of amyloid precursor protein (APP) by $\beta$ - and $\gamma$-secretases. SIRT1 had the surprising ability to activate the gene encoding the $\alpha$-secretase, which cleaves APP at a different location and directs the processing of APP away from the $\beta$ - and $\gamma$-secretases to reduce $A \beta$ production (Donmez et al. 2010). SIRT1 activated the $\alpha$-secretase gene $A D A M 10$ by deacetylating one of the transcription factors that binds to its promoter, the retinoic acid receptor $\beta$. The $\alpha$-secretase also cleaves the membrane protein Notch, involved in neurogenesis and neuroprotection, and this may be why SIRT1 evolved to regulate $A D A M 10$.

SIRT1 transgenic AD mice were protected from memory loss and plaques owing to protein aggregates of $A \beta$ in the brain. AD-affected patients also show $\mathrm{A} \beta$ plaques as well as a second protein aggregate: tangles of the Tau protein in neurons. Thus, $A \beta$ and Tau have been two leading candidates for the causal agents of AD. Remarkably, in a separate study in mice, SIRT1 was also demonstrated to deacetylate Tau protein to destabilize it and thereby reduce tangles (Min et al. 2010).

\section{MITOCHONDRIAL SIRTUINS}

Of the seven mammalian sirtuins, three are targeted to the mitochondrial matrix (Verdin et al. 2010), where they deacetylate or ADP-ribosylate enzymes to trigger metabolic adaptation to fuel sources such as amino acids and fatty acids during energy limitation.

\section{SIRT3 and Metabolism}

The major mitochondrial deacetylase SIRT3 promotes catabolism of acetate and fatty acids, as discussed above. Likewise, deacetylation of glutamate dehydrogenase $(\mathrm{GDH})$ allows amino acids to be converted into $\alpha \mathrm{KG}$ for metabolism. A recent proteomics screen identified many potential SIRT3 mitochondrial protein substrates, including ornithine transcarbamoylase (Hallows et al. 2011). Deacetylation of this urea cycle enzyme by SIRT3 activates the urea cycle for disposal of ammonia when amino acids are catabolically stripped of their carbon. The fact that SIRT5 was already known to deacetylate and activate another urea cycle enzyme, CPS1 (Nakagawa et al. 2009; Ogura et al. 2010), reinforces the importance of the urea cycle in sirtuin-mediated adaptations to energy limitation. SIRT3 was also shown to deacetylate and activate 3-hydroxy-3-methylglutaryl CoA synthase 2 (Hmgcs2), the rate-limiting enzyme for the synthesis of the ketone $\beta$-hydroxybuterate in the liver (Shimazu et al. 2010).

These metabolic adaptations all allow the organism to use fuels that might otherwise be stored (fat and amino acids) or ignored (acetate) and globally shift energy production away from carbohydrate catabolism. It was suggested that garnering energy from fat might per se reduce ROS production (Guarente 2008). This follows, because some of the electrons derived from oxidation of fatty acids feed into the electron transport chain via $\mathrm{FADH}_{2}$ (and not NADH), thus bypassing one of the sources of ROS production, complex I. Of course, this strategy does not bypass the other source of ROS production, complex III. However, the induction of SIRT3 might do so (see below).

\section{Mechanisms of Protection against Oxidative Damage by SIRT3}

Beyond these metabolic functions, SIRT3 has been shown to control the levels of ROS themselves by multiple mechanisms. The implications of these findings, if confirmed in a broad range of cell types, may redirect the focus of attention back to mitochondrial oxidative damage as a primary cause of aging, unite some of the central ideas on aging, and suggest novel ways to intervene pharmacologically.

Recent studies have revealed several antioxidant functions for SIRT3 in mitochondria. The study of Someya et al. (2010) discussed above proposed a specific mechanism by which SIRT3 protected cochlea cells of the inner ear against oxidative damage during CR. SIRT3 deacetylated and activated the TCA cycle enzyme isocitrate dehydrogenase 2 (IDH2), which produces NADPH in the mitochondria. The elevated NADPH, in turn, is necessary for glutathione reductase, which converts reduced oxidized glutathione disulfide (GSSG) into glutathione, the cofactor used by mitochondrial glutathione peroxidase (GPX) to detoxify ROS. Interestingly, another target of SIRT3, GDH (Lombard et al. 2007; Schlicker et al. 2008), also produces NADPH and may contribute to the increased pool of glutathione that is available for GPX to detoxify ROS within mitochondria.

Two other studies show that SIRT3 deacetylates the critical antioxidant enzyme manganese superoxide dismutase (SOD2) in the mitochondrial matrix (Qiu et al. 2010; Tao et al. 2010). Deacetylation of SOD2 resulted in increased specific activity and enhanced scavenging of ROS. Strikingly, the two studies revealed different lysines in SOD2 that were deacetylated by SIRT3 (K53 and K68 in the Qiu et al. study and K122 in the Tao et al. study). In both studies, mutating the lysine(s) to 
arginine gave rise to a hyperactive SOD2 that was not further enhanced by SIRT3, suggesting that all of these lysines may be important. The Qiu et al. study also showed that CR itself resulted in the deacetylation and activation of SOD2, presumably by up-regulating SirT3. This finding may help explain why CR has consistently been associated with a reduction in ROS levels in mitochondria (Weindruch and Walford 1988). It is important to note that the role of SIRT3 on ROS detoxification through the generation of NADPH and activation of SOD2 is confined to the mitochondrial matrix and does not represent a boost in antioxidant capacity in the nucleus or cytosol. However, SIRT3 does suppress ROS-responsive pathways that are induced by cardiac hypertrophy in the cytosol, such as RAS, AKT, and MAPK signaling (Sundaresan et al. 2009).

SIRT3 may do so by deacetylating numerous components of the electron transport chain itself. For example, SIRT3 was shown to deacetylate subunits of complex I (NADH dehydrogenase; Ahn et al. 2008) and complex II (succinate dehydrogenase; Cimen et al. 2009), whereas mitochondria from SIRT3 ${ }^{-/-}$MEFs had reduced complex I, II, and III activity (Ahn et al. 2008; Cimen et al. 2009; Kim et al. 2010). Importantly, SIRT3 suppressed ROS from complex III (Bell et al. 2011), which directs ROS to both the matrix and cytoplasm (Muller et al. 2004). Whereas SIRT3 has not been demonstrated to deacetylate subunits of the ATP generating complex V, it was shown to physically interact with complex V subunits (Law et al. 2009). The reduced steady-state ATP levels in SIRT3 ${ }^{-/-}$cells may result from a defect in deacetylating electron transport chain components (Ahn et al. 2008); i.e., SIRT3 deficiency may result in reduced efficiency of electron transfer within the cytochrome chain, thereby increasing the probability of electrons being transferred to molecular oxygen to generate ROS at the expense of ATP production. The fact that SIRT3 has apparently evolved multiple mechanisms to reduce ROS indicates a close relationship among this sirtuin, $\mathrm{CR}$, and aging.

Both SIRT3 and SIRT4 were shown to be necessary for nutrient-sensitive protection from genotoxic stress facilitated by increased mitochondrial $\mathrm{NAD}^{+}$levels (Yang et al. 2007). In addition, SIRT3 deacetylates cyclophilin $\mathrm{D}$, shown to inhibit apoptosis induced by the opening of the mitochondrial permeability transition pore (Hafner et al. 2010). Reduction of the ROS burden coupled with an increase in stress resistance may maintain cells, such as the cochlea cells of the inner ear, during CR.

\section{SIRT3, HIF-1 $\alpha$, and Cancer}

SIRT3 $^{-/-}$mice were found to develop mammary tumors at 24 months of age (Kim et al. 2010). Consistent with the idea that SirT3 is a tumor suppressor, SIRT3 ${ }^{-/-}$ mouse embryo fibroblasts (MEFs) were easier to transform (requiring a single oncogene) compared with wildtype MEFs (which also require inactivation of a tumor suppressor gene). SIRT3 ${ }^{-/-}$cells also showed increased superoxide levels and greater chromosome instability when stressed. All of these findings suggested that SIRT3 suppresses tumor initiation in vivo by preventing chromosomal instability.

Additional insight into the role of SIRT3 as a tumor suppressor came from two studies examining the relationship between SIRT3 and the transcription factor hypoxiainducible factor, HIF-1 $\alpha$. ROS-mediated stabilization of HIF-1 $\alpha$ is the major adaptive response to hypoxia. In one study, the inactivation of SIRT3 by short hairpin RNA (shRNA) in several tumor cell lines gave rise to higher ROS and HIF-1 $\alpha$ activation in normoxia and hyperactivation of HIF-1 $\alpha$ in hypoxic conditions. This is consistent with the idea that SIRT3 suppresses ROS, which are necessary for the hypoxic induction of HIF-1 $\alpha$. Moreover, transgenic overexpression of SIRT3 prevented the induction of HIF- $1 \alpha$ by hypoxia (Bell et al. 2011). This finding is interesting because HIF-1 $\alpha$ is known to enhance the growth of tumors by adapting them to hypoxic conditions that occur during tumor development (Majmundar et al. 2010). These adaptations include induction of aerobic glycolysis for biosynthetic intermediates (the Warburg effect; Vander Heiden et al. 2009) and induction of angiogenesis to bring additional glucose and oxygen to the tumor. In fact, xenografts of tumor cells with normal, reduced, or elevated SIRT3 levels were generated, and the tumors with reduced SirT3 were the largest, whereas the tumors with the overexpressed SIRT3 were the smallest (Bell et al. 2011). Interestingly, antioxidant treatment was able to normalize the size of the tumors lacking SirT3. Moreover, inducing SIRT3 expression after initiation of tumor formation sufficed to arrest tumor growth, suggesting that SIRT3 is important not only in the initiation of tumors but also in the maintenance and progression of tumors.

SIRT $^{-/-}$MEFs also were shown to consume more glucose, generate more lactate, and have higher levels of glycolytic intermediates and lower levels of some TCA cycle intermediates (Finley et al. 2011). These data are also consistent with altered cellular metabolism that is similar to the Warburg effect. Consistent with this metabolic reprogramming, transcriptional profiling of SIRT3 ${ }^{-/-}$MEFs demonstrated hyperactivation of HIF-1 $\alpha$ targets, which was reversed in the presence of shRNA's targeting of HIF-1 $\alpha$. Strikingly, overexpression of SIRT3 reversed the Warburg effect in different breast cancer cell lines. The above data demonstrate that SIRT3 may suppress the Warburg effect through the ROSHIF- $1 \alpha$ axis. In addition, the deacetylation of cyclophilin D by SIRT3 may contribute to this suppression because it was also shown to be essential for the dissociation of hexokinase II from mitochondria (Shulga et al. 2010), thereby decreasing the entry of glucose into glycolysis. It will be important to determine how generally SIRT3mediated control of ROS governs the Warburg effect in other kinds of tumors. Interestingly, SIRT1 was also shown to repress HIF- $1 \alpha$ by deacetylating it (Lim et al. 2010), illustrating a second target (along with NF-кB) in which two sirtuins work in concert.

It is amazing at this late date that data are first emerging linking SIRT3 gene deletion and reduction in protein 
levels to human breast and ovarian carcinomas (40\%) and other tumors $(20 \%-30 \%$; Kim et al. 2010; Finley et al. 2011). These findings suggest that SIRT3 is an important tumor suppressor in human cancers. Whereas it has been suggested that aging may be the price for zealous tumor suppression early in life (e.g., by p53; Tyner et al. 2002), the above findings suggest that the tumor suppressor SIRT3 involves no such tradeoff and actually links tumor suppression to a slowing of aging.

\section{Do SIRT1 and SIRT3 Cooperate in Calorie Restriction?}

SIRT1 and SIRT3 are required for the responses to CR and may be linked by one linear pathway. This follows because the coactivator PGC- $1 \alpha$ and the estrogen-related receptor $\alpha$ were shown to bind to the SIRT3 promoter to activate transcription (Kong et al. 2010). Because SIRT1 has been convincingly shown to deacetylate PGC- $1 \alpha$ to increase its potential to activate transcription (Nemoto et al. 2005; Rodgers et al. 2005), it is tempting to draw a linear pathway in which CR triggers an increase in SIRT1 activity, thereby activating SIRT3 expression via deacetylation of PGC- $1 \alpha$. This strategy would coordinate the CR response between the nucleus and the mitochondria and might be a core mechanism driving the antiaging effects of this dietary regimen.

\section{CONCLUSION}

Sirtuins are regulators of metabolism in response to diet. A growing body of evidence indicates that sirtuins thus slow aging and diseases of aging, although lifespan extension has not yet been observed. This latter finding may be because SIRT1 has so many important functions in mammalian physiology that global up-regulation can exert opposing effects. Alternatively, global up-regulation may indeed slow aging globally but not affect the proximal causes, for example, lymphoma, limiting life span in the mouse strains tested.

Knocking out the mitochondrial SIRT3 (Onyango et al. 2002; Schwer et al. 2002) causes no obvious phenotypes, at least in young mice (Lombard et al. 2007). However, more subtle analyses have shown that CR induces SIRT3 levels, resulting in lower ROS and oxidative damage to mitochondria. So far, most of the evidence linking SIRT3 to these processes involves loss of function, which triggers greater ROS and mitochondrial oxidative damage and loss of protection by CR. Cell-based studies have recently provided the first evidence that gain of function has the potential to exert opposite effects (Bell et al. 2011). It will be important to expand these studies by generating transgenic mice with increased SIRT3 expression.

SIRT3 is the only sirtuin for which a human polymorphism has been reported to associate with extreme longevity. This allele creates an enhancer in intron 5 of the SIRT3 gene and is highly enriched in a long-lived population in southern Italy (Rose et al. 2003; Bellizzi et al. 2005, 2009). It will be important to carry out comprehensive analyses of SIRT3 polymorphisms in other populations to confirm the link between SIRT3 and human longevity. More generally, genetic and physiological studies are now warranted to examine the relationship among SIRT1, and indeed all of the sirtuins, human aging, and disease.

\section{ACKNOWLEDGMENTS}

Work in my laboratory was supported by the Glenn Medical Foundation and the National Institutes of Health.

\section{REFERENCES}

Ahn BH, Kim HS, Song S, Lee IH, Liu J, Vassilopoulos A, Deng CX, Finkel T. 2008. A role for the mitochondrial deacetylase Sirt3 in regulating energy homeostasis. Proc Natl Acad Sci 105: $14447-14452$.

Bai P, Cantó C, Oudart H, Brunyánszki A, Cen Y, Thomas C, Yamamoto H, Huber A, Kiss B, Houtkooper RH, et al. 2011. PARP-1 inhibition increases mitochondrial metabolism through SIRT1 activation. Cell Metab 13: 461-468.

Banks AS, Kon N, Knight C, Matsumoto M, Gutiérrez-Juárez R, Rossetti L, Gu W, Accili D. 2008. SirT1 gain of function increases energy efficiency and prevents diabetes in mice. Cell Metab 8: 333-341.

Barger JL, Kayo T, Vann JM, Arias EB, Wang J, Hacker TA, Wang Y, Raederstorff D, Morrow JD, Leeuwenburgh C, et al. 2008. A low dose of dietary resveratrol partially mimics caloric restriction and retards aging parameters in mice. PLoS One 3: e2264.

Baur JA, Pearson KJ, Price NL, Jamieson HA, Lerin C, Kalra A, Prabhu VV, Allard JS, Lopez-Lluch G, Lewis K, et al. 2006. Resveratrol improves health and survival of mice on a highcalorie diet. Nature 444: 337-342.

Bauer JH, Morris SN, Chang C, Flatt T, Wood JG, Helfand SL. 2009. dSir2 and Dmp53 interact to mediate aspects of CR-dependent lifespan extension in D. melanogaster. Aging (Albany NY) 1: 38-48.

Bell EL, Guarente L. 2011. The SirT3 divining rod points to oxidative stress. Mol Cell 42: 561-568.

Bell EL, Emerling BM, Ricoult SJH, Guarente L. 2011. SirT3 suppresses hypoxia inducible factor $1 \alpha$ and tumor growth by inhibiting mitochondrial ROS production. Oncogene 30: 2986-2996.

Bellizzi D, Rose G, Cavalcante P, Covello G, Dato S, De Rango F, Greco V, Maggiolini M, Feraco E, Mari V, et al. 2005. A novel VNTR enhancer within the SIRT3 gene, a human homologue of SIR2, is associated with survival at oldest ages. Genomics 85: 258-263.

Bellizzi D, Covello G, Di Cianni F, Tong Q, De Benedictis G. 2009. Identification of GATA2 and AP-1 Activator elements within the enhancer VNTR occurring in intron 5 of the human SIRT3 gene. Mol Cells 28: 87-92.

Berdichevsky A, Viswanathan M, Horvitz R, Guarente L. 2006. C. elegans SIR-2.1 interacts with $14-3-3$ proteins to activate DAF-16 and extend lifespan. Cell 16: 1165-1178.

Birkenfield AL, Lee HY, Guebre-Egziabher F, Alves TC, Jurczak MJ, Jornayvaz FR, Zhang D, Hsiao JJ, Martin-Montalvo A, Fischer-Rosinsky A, et al. 2011. Deletion of mammalian INDY homolog mimics aspects of dietary restriction and protects against adiposity and insulin resistance in mice. Cell Metab 14: 184-195.

Boily G, Seifert EL, Bevilacqua L, He XH, Sabourin G, Estey C, Moffat C, Crawford S, Saliba S, Jardine K, et al. 2008. SirT1 regulates energy metabolism and response to caloric restriction in mice. PLoS One 3: e1759.

Boily G, He XH, Pearce B, Jardine K, McBurney MW. 2009. SirT1-null mice develop tumors at normal rates but are poorly protected by resveratrol. Oncogene 28: 2882-2893. 
Bordone L, Cohen D, Robinson A, Motta MC, van Veen E, Czopik A, Steele AD, Crowe H, Marmor S, Luo J, et al. 2007. SIRT1 transgenic mice show phenotypes resembling calorie restriction. Aging Cell 6: 759-767.

Chen D, Steele AD, Lindquist S, Guarente L. 2005. Increase in activity during calorie restriction requires Sirt1. Science 310: 1641.

Chen D, Bruno J, Easlon E, Lin SJ, Cheng HL, Alt FW, Guarente L. 2008. Tissue-specific regulation of SIRT1 by calorie restriction. Genes Dev 22: 1753-1757.

Cimen H, Han M-J, Yang Y, Tong Q, Koc H, Koc EC. 2009. Regulation of succinate dehydrogenase activity by SIRT3 in mammalian mitochondria. Biochemistry 49: 304-311.

Cohen HY, Miller C, Bitterman KJ, Wall NR, Hekking B, Kessler B, Howitz KT, Gorospe M, de Cabo R, Sinclair DA. 2004. Calorie restriction promotes mammalian cell survival by inducing the SIRT1 deacetylase. Science 305: 390-392.

Cohen DE, Supinski AM, Bonkowski MS, Donmez G, Guarente LP. 2009. Neuronal SIRT1 regulates endocrine and behavioral responses to calorie restriction. Genes Dev 23: $2812-$ 2817.

Dai H, Kustigian L, Carney D, Case A, Considine T, Hubbard BP, Perni RB, Riera TV, Szczepankiewicz B, Vlasuk GP, Stein RL. 2010. SIRT1 activation by small molecules: Kinetic and biophysical evidence for direct interaction of enzyme and activator. J Biol Chem 285: 32695-32703.

Donmez G, Wang D, Cohen DE, Guarente L. 2010. SIRT1 suppresses $\beta$-amyloid production by activating the $\alpha$-secretase gene ADAM10. Cell 142: 320-332.

Finkel T, Deng CX, Mostoslavsky R. 2009. Recent progress in the biology and physiology of sirtuins. Nature 460: 587-591.

Finley LW, Carracedo A, Lee J, Souza A, Egia A, Zhang J, Teruya-Feldstein J, Moreira PI, Cardoso SM, Clish CB, et al. 2011. SIRT3 opposes reprogramming of cancer cell metabolism through HIF1 $\alpha$ destabilization Cancer Cell 19: 416428.

Firestein R, Blander G, Michan S, Oberdoerffer P, Ogino S, Campbell J, Bhimavarapu A, Luikenhuis S, de Cabo R, Fuchs C, et al. 2008. The SIRT1 deacetylase suppresses intestinal tumorigenesis and colon cancer growth. PLoS One 3: e2020.

Guarente L. 2008. Mitochondria-A nexus for aging, calorie restriction, and sirtuins? Cell 132: 171-176.

Guarente L. 2011. Sirtuins, aging, and medicine. New Engl J Med 364: 2235-2244.

Hafner AV, Dai J, Gomes AP, Xiao CY, Palmeira CM, Rosenzweig A, Sinclair DA. 2010. Regulation of the mPTP by SIRT3-mediated deacetylation of CypD at lysine 166 suppresses age-related cardiac hypertrophy. Aging 2: 914923.

Haigis MC, Sinclair DA. 2010. Mammalian sirtuins: Biological insights and disease relevance. Annu Rev Pathol 5: 253295.

Haigis M, Mostoslavsky R, Haigis K, Christodolou D, Murphy A, Valenzuala D, Yancopoulos G, Karow M, Blander G, Weindruch R, et al. 2006. SIRT4 regulates glutamate dehydrogenase and insulin secretion in pancreatic $\beta$ cells. Cell 126: $941-954$.

Hallows WC, Lee S, Denu JM. 2006. Sirtuins deacetylate and activate mammalian acetyl-CoA synthetases. Proc Natl Acad Sci 103: 10230-10235.

Hallows WC, Yu W, Smith BC, Devries MK, Ellinger JJ, Someya S, Shortreed MR, Prolla T, Markley JL, Smith LM, et al. 2011. Sirt3 promotes the urea cycle and fatty acid oxidation during dietary restriction. Mol Cell 41: 139-149.

Herranz D, Muñoz-Martin M, Cañamero M, Mulero F, Martinez-Pastor B, Fernandez-Capetillo O, Serrano M. 2010. Sirt1 improves healthy ageing and protects from metabolic syndrome-associated cancer. Nat Commun 1: 3 .

Hirschey MD, Shimazu T, Goetzman E, Jing E, Schwer B, Lombard DB, Grueter CA, Harris C, Biddinger S, Ilkayeva OR, et al. 2010. SIRT3 regulates mitochondrial fatty-acid oxidation by reversible enzyme deacetylation. Nature 464: $121-125$.
Howitz KT, Bitterman KJ, Cohen HY, Lamming DW, Lavu S, Wood JG, Zipkin RE, Chung P, Kisielewski A, Zhang LL, et al. 2003. Small molecule activators of sirtuins extend Saccharomyces cerevisiae lifespan. Nature 425: 191-196.

Imai S, Guarente L. 2010. Ten years of NAD-dependent SIR2 family deacetylases: Implications for metabolic diseases. Trends Pharm Sci 31: 212-220.

Imai S, Armstrong CM, Kaeberlein M, Guarente L. 2000. Transcriptional silencing and longevity protein Sir2 is an NADdependent histone deacetylase. Nature 403: 795-800.

Kaeberlein M, McVey M, Guarente L. 1999. The SIR2/3/4 complex and SIR2 alone promote longevity in S. cerevisiae by two different mechanisms. Genes Dev 13: 2570-2581.

Kawahara TL, Michishita E, Adler AS, Damian M, Berber E, Lin M, McCord RA, Ongaigui KC, Boxer LD, Chang HY, Chua KF. 2009. SIRT6 links histone H3 lysine 9 deacetylation to NF- $\mathrm{BB}$-dependent gene expression and organismal life span. Cell 136: 62-74.

Kim SC, Sprung R, Chen Y, Xu Y, Ball H, Pei J, Cheng T, Kho Y, Xiao H, Xiao L, et al. 2006. Substrate and functional diversity of lysine acetylation revealed by a proteomics survey. Mol Cell 4: 607-618.

Kim D, Nguyen MD, Dobbin MM, Fischer A, Sananbenesi F, Rodgers JT, Delalle I, Baur JA, Sui G, Amour SM, et al. 2007. SIRT1 deacetylase protects against neurodegeneration in models for Alzheimer's disease and amyotrophic lateral sclerosis. EMBO J 26: 3169-3179.

Kim HS, Patel K, Muldoon-Jacobs K, Bisht KS, Aykin-Burns N, Pennington JD, van der Meer R, Nguyen P, Savage J, Owens $\mathrm{KM}$, et al. 2010. SIRT3 is a mitochondria-localized tumor suppressor required for maintenance of mitochondrial integrity and metabolism during stress. Cancer Cell 17: $41-52$.

Klar AJ, Fogel S, Macleod K. 1979. MAR1-A regulator of the $\mathrm{HMa}$ and $\mathrm{HM} \alpha$ loci in Saccharomyces cerevisiae. Genetics 93: $37-50$.

Kong X, Wang R, Xue Y, Liu X, Zhang H, Chen Y, Fang F, Chang Y. 2010. Sirtuin 3, a new target of PGC-1 $\alpha$, plays an important role in the suppression of ROS and mitochondrial biogenesis. PloS One 5: e11707.

Lagouge M, Argmann C, Gerhart-Hines Z, Meziane H, Lerin C, Daussin F, Messadeq N, Milne J, Lambert P, Geny B, et al. 2006. Resveratrol improves mitochondrial function and protects against metabolic disease by activating SIRT1 and

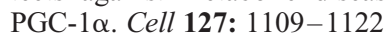

Law IKM, Liu L, Xu A, Lam KSL, Vanhoutte PM, Che C-M, Leung PTY, Wang Y. 2009. Identification and characterization of proteins interacting with SIRT1 and SIRT3: Implications in the anti-aging and metabolic effects of sirtuins. Proteomics 9: 2444-2456.

Lim JH, Lee YM, Chun YS, Chen J, Kim JE, Park JW. 2010. Sirtuin 1 modulates cellular responses to hypoxia by deacetylating hypoxia-inducible factor $1 \alpha$. Mol Cell 38: $864-878$.

Lui Y, Denton R, Chen D, Hedrick S, Ravnskjaer K, Schenk S, Milne J, Meyers DJ, Cole P, Yates J III, et al. 2008. A fasting inducible switch modulates gluconeogenesis via activator/ coactivator exchange. Nature 456: 269-273.

Lombard DB, Alt FW, Cheng HL, Bunkenborg J, Streeper RS, Mostoslavsky R, Kim J, Yancopoulos G, Valenzuela D, Murphy A, et al. 2007. Mammalian Sir2 homolog SIRT3 regulates global mitochondrial lysine acetylation. Mol Cell Biol 27: 8807-8814.

Majmundar AJ, Wong WJ, Simon MC. 2010. Hypoxia-inducible factors and the response to hypoxic stress. Mol Cell 40: 294-309.

Milne JC, Lambert PD, Schenk S, Carney DP, Smith JJ, Gagne DJ, Jin L, Boss O, Perni RB, Vu CB, et al. 2007. Small molecule activators of SIRT1 as therapeutics for the treatment of type 2 diabetes. Nature 450: 712-716.

Min SW, Cho SH, Zhou Y, Schroeder S, Haroutunian V, Seeley WW, Huang EJ, Shen Y, Masliah E, Mukherjee C, et al. 2010. Acetylation of tau inhibits its degradation and contributes to tauopathy. Neuron 67: 953-966. 
Minor RK, Baur JA, Gomes AP, Ward TM, Csiszar A, Mercken EM, Abdelmohsen K, Shin Y-K, Canto C, Scheibye-Knudsen M, et al. 2011. SRT1720 improves survival and healthspan of obese mice. Sci Rep 1: 70.

Muller FL, Liu Y, Van Remmen H. 2004. Complex III releases superoxide to both sides of the inner mitochondrial membrane. J Biol Chem 279: 49064-49073.

Nakagawa T, Guarente L. 2011. Sirtuins at a glance. J Cell Sci 124(Pt 6): 833-838.

Nakagawa T, Haigis M, Lomb D, Guarente L. 2009. SIRT5 Deacetylates carbamoyl phosphate synthetase 1 and regulates the urea cycle. Cell 137: 560-570.

Nemoto S, Fergusson MM, Finkel T. 2005. SIRT1 functionally interacts with the metabolic regulator and transcriptional coactivator PGC-1 $\alpha$. J Biol Chem 280: 16456-16460.

Nisoli E, Tonello C, Cardile A, Cozzi V, Bracale R, Tedesco L, Falcone S, Valerio A, Cantoni O, Clementi E, et al. 2005. Calorie restriction promotes mitochondrial biogenesis by inducing the expression of eNOS. Science 310: 314-317.

Noriega LG, Feige JN, Canto C, Yamamoto H, Yu J, Herman MA, Mataki C, Kahn BB, Auwerx J. 2011. CREB and ChREBP oppositely regulate SIRT1 expression in response to energy availability. EMBO Rep doi: $10.1038 /$ embor. 2011.151.

Ogura M, Nakamura Y, Tanaka D, Zhuang X, Fujita Y, Obara A, Hamasaki A, Hosokawa M, Inagaki N. 2010. Overexpression of SIRT5 confirms its involvement in deacetylation and activation of carbamoyl phosphate synthetase 1. Biochem Biophys Res Commun 393: 73-78.

Onyango P, Celic I, McCaffery JM, Boeke JD, Feinberg AP. 2002. SIRT3, a human SIR2 homologue, is an NAD-dependent deacetylase localized to mitochondria. Proc Natl Acad Sci 99: $13653-13658$.

Pacholec M, Bleasdale JE, Chrunyk B, Cunningham D, Flynn D, Garofalo RS, Griffith D, Griffor M, Loulakis P, Pabst B, et al. 2010. SRT1720, SRT2183, SRT1460, and resveratrol are not direct activators of SIRT1. J Biol Chem 285: 8340 8351.

Pfluger PT, Herranz D, Velasco-Miguel S, Serrano M, Tschöp MH. 2008. Sirt1 protects against high-fat diet-induced metabolic damage. Proc Natl Acad Sci 105: 9793-9798.

Picard F, Kurtev M, Chung N, Topark-Ngarm A, Senawong T, Machado De Oliveira R, Leid M, McBurney MW, Guarente L. 2004. Sirt1 promotes fat mobilization in white adipocytes by repressing PPAR- $\gamma$. Nature 429: 771-776.

Purushotham A, Schug TT, Xu Q, Surapureddi S, Guo X, Li X. 2009. Hepatocyte-specific deletion of SIRT1 alters fatty acid metabolism and results in hepatic steatosis and inflammation. Cell Metab 9: 327-338.

Qin W, Yang T, Ho L, Zhao Z, Wang J, Chen L, Zhao W, Thiyagarajan M, MacGrogan D, Rodgers JT, et al. 2006. Neuronal SIRT1 activation as a novel mechanism underlying the prevention of Alzheimer disease amyloid neuropathology by calorie restriction. J Biol Chem 281: 21745-21754.

Qiu X, Brown K, Hirschey MD, Verdin E, Chen D. 2010. Calorie restriction reduces oxidative stress by SIRT3-mediated SOD2 activation. Cell Metab 12: 662-667.

Rizki G, Iwata TN, Li J, Riedel CG, Picard CL, Jan M, Murphy CT, Lee SS. 2011. The evolutionarily conserved longevity determinants HCF-1 and SIR-2.1/SIRT1 collaborate to regulate DAF-16/FOXO. PLoS Genet 7: E1002235.

Rodgers JT, Lerin C, Haas W, Gygi SP, Spiegelman BM, Puigserver P. 2005. Nutrient control of glucose homeostasis through a complex of PGC-1 $\alpha$ and SIRT1. Nature 434: 113 118.

Rogina B, Helfand SL. 2004. Sir2 mediates longevity in the fly through a pathway related to calorie restriction. Proc Natl Acad Sci 101: 15998-16003.

Rose G, Dato S, Altomare K, Bellizzi D, Garasto S, Greco V, Passarino G, Feraco E, Mari V, Barbi C, et al. 2003. Variability of the SIRT3 gene, human silent information regulator Sir2 homologue, and survivorship in the elderly. Exp Gerontol 38: $1065-1070$.
Schlicker C, Gertz M, Papatheodorou P, Kachholz B, Becker CFW, Steegborn C. 2008. Substrates and Regulation Mechanisms for the Human Mitochondrial Sirtuins Sirt3 and Sirt5. J Mol Biol 382: 790-801.

Schwer B, North BJ, Frye RA, Ott M, Verdin E. 2002. The human silent information regulator (Sir)2 homologue hSIRT3 is a mitochondrial nicotinamide adenine dinucleotide-dependent deacetylase. J Cell Biol 158: 647-657.

Schwer B, Bunkenborg J, Verdin RO, Andersen JS, Verdin E. 2006. Reversible lysine acetylation controls the activity of the mitochondrial enzyme acetyl-CoA synthetase 2. Proc Natl Acad Sci 103: 10224-10229.

Schwer B, Eckersdorff M, Li Y, Silva JC, Fermin D, Kurtev MV, Giallourakis C, Comb MJ, Alt FW, Lombard D. 2009. Calorie restriction alters mitochondrial protein acetylation. Aging Cell 5: 604-606.

Shimazu T, Hirschey MD, Hua L, Dittenhafer-Reed KE, Schwer B, Lombard DB, Li Y, Bunkenborg J, Alt FW, Denu JM, et al. 2010. SIRT3 deacetylates mitochondrial 3-hydroxy-3-methylglutaryl CoA synthase 2 and regulates ketone body production. Cell Metab 12: 654-661.

Shulga N, Wilson-Smith R, Pastorino JG. 2010. Sirtuin-3 deacetylation of cyclophilin D induces dissociation of hexokinase II from the mitochondria. J Cell Sci 123: 894-902.

Someya S, Yu W, Hallows WC, Xu J, Vann JM, Leeuwenburgh C, Tanokura M, Denu JM, Prolla TA. 2010. Sirt3 mediates reduction of oxidative damage and prevention of age-related hearing loss under caloric restriction. Cell 143: 802-812.

Sundaresan NR, Gupta M, Kim G, Rajamohan SB, Isbatan A, Gupta MP. 2009. Sirt3 blocks the cardiac hypertrophic response by augmenting Foxo3a-dependent antioxidant defense mechanisms in mice. $J$ Clin Invest 119: 2758-2771.

Tanner KG, Landry J, Sternglanz R, Denu JM. 2000. Silent information regulator 2 family of NAD-dependent histone/ protein deacetylases generates a unique product, 1-O-acetylADP-ribose. Proc Natl Acad Sci 97: 14178-14182.

Tao R, Coleman MC, Pennington JD, Ozden O, Park S-H, Jiang H, Kim H-S, Flynn CR, Hill S, Hayes McDonald W, et al. 2010. Sirt3-mediated deacetylation of evolutionarily conserved lysine 122 regulates MnSOD activity in response to stress. Mol Cell 40: 893-904.

Tissenbaum HA, Guarente L. 2001. Increased dosage of a sir-2 gene extends lifespan in Caenorhabditis elegans. Nature 410: 227-230.

Tyner SD, Venkatachalam S, Choi J, Jones S, Ghebranious N, Igelmann H, Lu X, Soron G, Cooper B, Brayton C, et al. 2002. p53 Mutant mice that display early ageing-associated phenotypes. Nature 415: 45-53.

Vander Heiden MG, Cantley LC, Thompson CB. 2009. Understanding the Warburg effect: The metabolic requirements of cell proliferation. Science 324: 1029-1033.

Verdin E, Hirschey MD, Finley LW, Haigis MC. 2010. Sirtuin regulation of mitochondria: Energy production, apoptosis, and signaling. Trends Biochem Sci 35: 669-675.

Viswanathan M, Guarente L. 2011. Regulation of Caenorhabditis elegans lifespan by sir-2.1 transgenes. Nature 477: E1 -E2.

Viswanathan M, Kim S, Berdichevsky A, Guarente L. 2005. A role for sir-2.1 regulation of ER stress response genes in determining C. elegans lifespan. Dev Cell 5: 605-616.

Wang RH, Zheng Y, Kim HS, Xu X, Cao L, Luhasen T, Lee MH, Xiao C, Vassilopoulos A, Chen W, et al. 2008. Interplay among BRCA1, SIRT1, and Survivin during BRCA1-associated tumorigenesis. Mol Cell 32: 11-20.

Wang Q, Zhang Y, Yang C, Xiong H, Lin Y, Yao J, Li H, Xie L, Zhao W, Yao Y. 2010. Acetylation of metabolic enzymes coordinates carbon source utilization and metabolic flux. Science 327: 1004-1007.

Weindruch R, Walford RL. 1988. The Retardation of Aging and Disease by Dietary Restriction. Thomas Charles C, Springfield, IL.

Wellen KE, Hatzivassiliou G, Sachdeva UM, Bui TV, Cross JR, Thompson CB. 2009. ATP-citrate lyase links cellular metabolism to histone acetylation. Science 324: 1076-1080. 
Westerheide SD, Anckar J, Stevens SM Jr, Sistonen L, Morimoto RI. 2009. Stress-inducible regulation of heat shock factor 1 by the deacetylase SIRT1. Science 323: $1063-$ 1066.

Xiong S, Salazar G, Patrushev N, Alexander RW. 2011. FoxO1 mediates an autofeedback loop regulating SIRT1 expression. J Biol Chem 286: 5289-5299.

Yang H, Yang T, Baur JA, Perez E, Matsui T, Carmona JJ, Lamming DW, Souza-Pinto NC, Bohr VA, Rosenzweig A, et al.
2007. Nutrient-sensitive mitochondrial $\mathrm{NAD}^{+}$levels dictate cell survival. Cell 130: 1095-1107.

Yeung F, Hoberg JE, Ramsey CS, Keller MD, Jones DR, Frye RA, Mayo MW. 2004. Modulation of NF-кB-dependent transcription and cell survival by the SIRT1 deacetylase. EMBOJ 23: $2369-2380$.

Zhao S, Xu W, Jiang W, Yu W, Lin Y, Zhang T, Yao J, Zhou L, Zeng Y, Li H, et al. 2010. Regulation of cellular metabolism by protein lysine acetylation. Science 327: 1000-1004. 


\section{$\$_{\text {CSH }}^{\infty}$ Cold Spring Harbor Symposia SYMPOSIA On Quantitative Biology}

\section{Sirtuins, Aging, and Metabolism}

Leonard Guarente

Cold Spring Harb Symp Quant Biol 2011 76: 81-90 originally published online November 23, 2011

Access the most recent version at doi:10.1101/sqb.2011.76.010629

References This article cites 95 articles, 28 of which can be accessed free at: http://symposium.cshlp.org/content/76/81.full.html\#ref-list-1

\section{License}

Email Alerting

Receive free email alerts when new articles cite this article - sign up in

Service the box at the top right corner of the article or click here. 